\title{
Hyperglycemia primes NETosis, which exacerbates ischemic brain damage
}

\section{Jiangshan Deng ( $\nabla$ johnson120@126.com)}

Shanghai Jiao Tong University Affiliated Six People's Hospital https://orcid.org/0000-0002-2996-5979

\section{Fei Zhao}

Shanghai Jiao Tong University Affiliated Six People's Hospital

\section{Yunlong Zhang}

Shanghai Jiao Tong University Affiliated Six People's Hospital

\section{Yajun Zhou}

Shanghai Jiao Tong University Affiliated Six People's Hospital

\section{Xiaofeng Xu}

Shanghai Jiao Tong University Affiliated Six People's Hospital

\section{Xiaojie Zhang}

Shanghai Jiao Tong University Affiliated Sixth People's Hospital

\section{Feng Wang}

Shanghai Jiao Tong University Affiliated Sixth People's Hospital

\section{Yuwu Zhao}

Shanghai Jiao Tong University Affiliated Sixth People's Hospital

\section{Research}

Keywords: middle cerebral artery infarction, diabetes, hyperglycemia, neutrophil extracellular traps

Posted Date: January 24th, 2020

DOI: https://doi.org/10.21203/rs.2.18044/v2

License: (c) (1) This work is licensed under a Creative Commons Attribution 4.0 International License.

Read Full License 
The authors have withdrawn this preprint from Research Square 